\title{
Re: Time to consider integration of a formal robotic-assisted surgical training program
}

\author{
Noah E. Canvasser ${ }^{1} \cdot$ Jeffrey Gahan ${ }^{2} \cdot$ Igor Sorokin $^{2}$
}

Published online: 1 March 2018

๑) Springer-Verlag London Ltd., part of Springer Nature 2018

Keywords Robotic surgery $\cdot$ Training $\cdot$ Curriculum

In the second Journal of Robotic Surgery (JORS) Twitter ${ }^{\mathrm{TM}}$ journal club, we discussed Vetter and colleague's manuscript regarding survey results of robotic surgical training for obstetric and gynecology residents in the United States [1]. In this study, 177 residents completed a 31-item questionnaire that covered a range of topics surrounding robotic training. Surprisingly, over 30\% of respondents did not need to complete a robotics training course prior to working on the console, and $50 \%$ did not have formal evaluations during their robotic training, When asked what area of robotic training was most and least useful, 50\% thought that the dual console was the most useful and 50\% thought that online modules were least useful. Personal time was often stated as a major limitation to robotic training, given most residents are not allotted robotic training time during a normal workweek. Discussion ensued (see supplementary Table 1).

There was consensus that formal robotic surgery curriculum is absolutely necessary. Because there is significant variation between centers (1) any training curriculum should not focus on a specific simulator, robot, or model (2), as mentioned in a review of robotic training curriculum [2].

Virtual simulators, as well as dry and wet lab environments (3), are necessary to teach residents to use the pedals, clutch, and drive the camera (4), but allowing residents to use such modalities requires specific curriculum and

Electronic supplementary material The online version of this article (https://doi.org/10.1007/s11701-018-0794-5) contains supplementary material, which is available to authorized users.

Noah E. Canvasser

ncanvasser@ucdavis.edu

1 Department of Urology, UC Davis Medical Center, 4860 Y Street, Suite 3500, Sacramento, CA 95817, USA

2 Department of Urology, UT Southwestern Medical Center, Dallas, TX, USA direction (5). In addition, trainees need formal evaluations of their simulation progress (6). Because access to simulation centers often does not align with hours residents are free (7), formal evaluations from faculty can be difficult to obtain. Video recording of simulator work is one potential solution (8), but this might not capture true hand technique at the console (9). Live mentoring with both trainee and faculty in a simulation center would allow more opportunities to correct trainees, as well as allow trainees a closer opportunity to watch how faculty operate on a simulator up close (10).

These sentiments were echoed in the first poll of the discussion (Fig. 1), where the majority of respondents felt live faculty presence was the best way to evaluate trainees completing a robotic training curriculum.

However, downsides to simulation were also mentioned. For example, digital simulators are lacking with tissue handling and dissection (11) and there is limited access to animal and cadaver simulation (12). Dry labs might be a more ideal setting for practice, as suturing is more realistic, plus there are models that allow dissection practice (13).

Another area of debate was resident exposure to bedside assisting during robotic surgery. In the second poll of the journal club, the majority of respondents $(73 \%)$ felt that trainees need to do at least 10 cases as a bedside assistant (Fig. 2). Bedside assisting gives a thorough exposure to robotic arm mechanics and function, and is sometimes a trainee's first exposure to laparoscopy (14-15). Some residents might view the bedside assist as a menial task, however, it is an invaluable experience that ultimately makes a better robotic surgeon (16). And, while the quantity of cases at the bedside might not be the most important metric (17), achieving competence at the bedside is a must (18).

Ultimately, a multi-modality curriculum, with live simulation and timely feedback by faculty, are important to ensure trainees receive appropriate robotics exposure prior 
J Robotic Surgery

@JRobotSurg

Twitter Journal club discussion happening now. What is the most practical way to evaluate skills of residents completing a robotic training curriculum? \#robojc

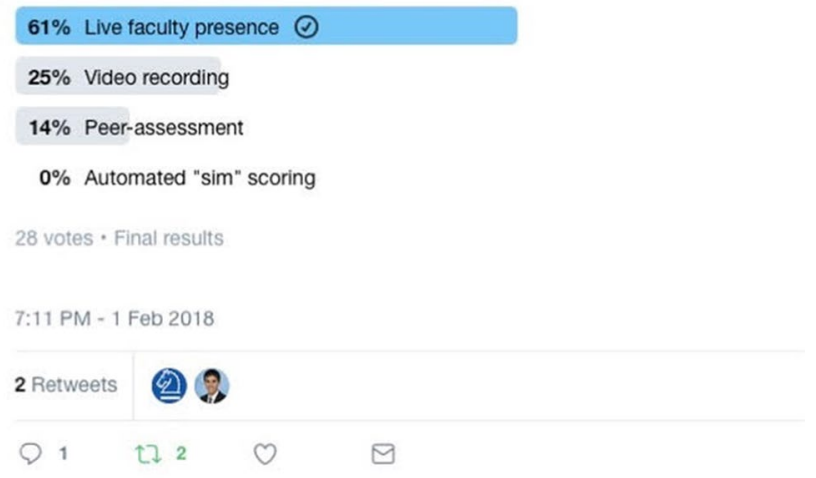

Fig. 1 First Twitter ${ }^{\mathrm{TM}}$ poll of discussion highlighting ways to evaluate residents robotic skills during training curriculum

J Robotic Surgery

QJRobotSurg

How many cases should residents bedside assist on the robot before they sit at the console? \#robojc

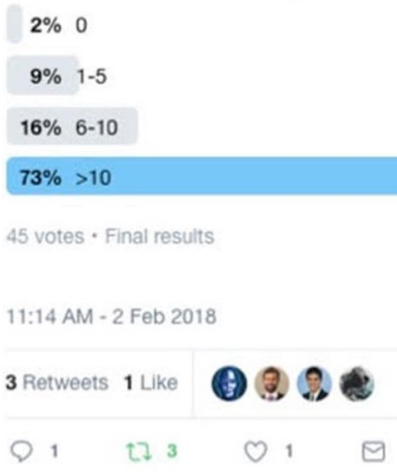

Fig. 2 Second Twitter ${ }^{\mathrm{TM}}$ poll of discussion emphasizing use of bedside assisting prior to console exposure to performing live surgery, as well as throughout their years of training (19).

\section{Compliance with ethical standards}

Conflict of interest Noah Canvasser, Jeffrey Gahan, and Igor Sorokin declare that they have no conflict of interest.

Ethical approval This article does not contain any studies with human participants or animals performed by any of the authors.

\section{References}

1. Vetter MH, Palettas M, Hade E et al (2017) Time to consider integration of a formal robotic-assisted surgical training program into obstetrics/gynecology residency curricula. J Robot Surg. https ://doi.org/10.1007/s11701-017-0775-0

2. Fisher RA, Dasgupta P, Mottrie A et al (2015) An over-view of robot assisted surgery curricula and the status of their validation. Int J Surg 13:115-123. https://doi.org/10.1016/j.jisu.2014.11.033 